\title{
Usefulness of Thermoluminescent Slab Dosimeter for Postal Dosimetry Audit of External Radiotherapy Systems
}

\author{
Daiki Maruyama, ${ }^{1 *}$ Shin Yanagisawa, ${ }^{1}$ Yusuke Koba, ${ }^{2}$ \\ Takayuki Andou, ${ }^{3}$ and Kiyomitsu Shinsho ${ }^{1}$ \\ ${ }^{1}$ Tokyo Metropolitan University,7-2-10 Higashi-Oku, Arakawa-ku, Tokyo 116-8551, Japan \\ ${ }^{2}$ QST NIRS, 4-9-1 Anagawa, Inage-ku, Chiba-shi, Chiba 263-8555, Japan \\ ${ }^{3}$ Chiba Ceramic MFG Co., Ltd., 2-10-14 Inage-higashi,Inage-ku, Chiba-shi, Chiba 263-0031, Japan
}

(Received November 5, 2019; accepted February 3, 2020)

Keywords: TLD, radiotherapy, postal dosimetry, audit, quality assurance

In this study, the feasibility of using a thermoluminescent slab dosimeter (TLSD) in postal dosimetry audit for radiotherapy systems is examined. First, changes in material structure are evaluated by measuring changes in TLSD weight and temperature after heating and cooling. Then, a fading correction factor is derived by measuring the relationship between the elapsed time after irradiation and thermoluminescence (TL) intensity. Finally, the uncertainty in the absorbed dosimetry using the TLSD with the TL absorbed dose correction factor is evaluated. It is shown that the TLSD weight and heat flow change after heating. These changes are reversible upon cooling. Therefore, the material structure of the TLSD does not change after heating. The fading effect for the TLSD can be minimized by setting the elapsed time to more than $100 \mathrm{~h}$. Furthermore, measurement accuracy can be improved by correcting the fading. The overall measurement uncertainty or expanded relative standard uncertainty for this measurement method is $1.80 \%$. The TLSD has high material stability and measurement accuracy under a certain condition equivalent to that of conventional postal dosimetry audit conducted using a glass dosimeter. The TLSD can be used as a 2D measurement device for postal dosimetry audit.

\section{Introduction}

Radiation treatment planning systems (TPSs) can accurately identify normal tissues and a disease site on the basis of computed tomography images. The treatment plan concentrates the radiation beam at the disease site. Setup accuracy (via patient immobilization) and individual patient dose quality assurance testing (carried out using various radiation detectors) are necessary for delivering radiation to a patient according to a treatment plan. ${ }^{(1)}$

For dose distribution verification, the output dose accuracy of a high-energy photon beam is crucial. The output dose is the absorbed dose output from the radiotherapy system calculated using a TPS. Output dose evaluation is performed by each facility using an ionization chamber

*Corresponding author: e-mail: maruyama_daiki@med.jrc.or.jp

https://doi.org/10.18494/SAM.2020.2697 
dosimeter with traceable dose measurement. However, human error cannot be eliminated in such evaluations. Output dose measurement by third parties has recently increased in importance as highly objective quality control.

A representative third-party output dose evaluation method is the TLD Postal Dose Audit Service, which uses a thermoluminescent dosimeter (TLD), jointly implemented by the International Atomic Energy Agency and the World Health Organization since 1969. ${ }^{(2,3)}$ In this method, the TLD is transported to the facility and irradiated by the facility staff according to the protocol. The irradiated TLD is returned to the evaluation organization and the absorbed dose is measured and evaluated. Third-party evaluation in Japan has been carried out since 2007 under the Radiotherapy Output Dose Measurement Project, which uses a glass dosimeter, by the Association for Nuclear Technology in Medicine. ${ }^{(4)}$ Both evaluation methods are performed using absorbed dosimetry at a reference depth on the central axis of the beam. A glass dosimeter can be read repeatedly and has a negligible fading effect. These properties are useful for postal dosimetry audit. ${ }^{(5)}$ There is thus potential for the combination of glass dosimetry and film dosimetry to become a tool for 2D dosimetry in postal audit.

Beam profile constancy is as important as output constancy for obtaining a complex dose distribution. Planar detectors can measure a 2D dose distribution. Although evaluating the beam profile is important for the quality control of a radiotherapy system, third-party evaluations consider only point doses, in both Japan and abroad. ${ }^{(2-4,6)}$ It is thus desirable to build a system capable of performing third-party evaluations on more items than currently possible.

We are developing a thermoluminescent slab dosimeter (TLSD) based on the thermoluminescence (TL) properties of a Cr-doped $\mathrm{Al}_{2} \mathrm{O}_{3}$ ceramic plate. ${ }^{(7)}$ A TLSD has been shown to be a high-resolution detector with an image pixel size of $0.05 \mathrm{~mm}$ and a TL reproducibility of about $1 \%$. It has been shown that the dose distribution can be obtained using the absorbed dose correction factor. ${ }^{(8)}$ If a sufficiently high accuracy in postal dosimetry measurement using a TLSD can be achieved, TLSDs can be used by third parties to evaluate dose distributions.

A previous study showed that the energy, field size, and water depth dependences of TLSDs are different from those for ionization chamber measurement. ${ }^{(9)}$ The response ratio decreases by approximately $8 \%$ for a square field with $3 \mathrm{~cm}$ sides compared with that for a square field with $10 \mathrm{~cm}$ sides. In addition, the TL response ratio showed over-response at a depth of $2 \mathrm{~cm}$ compared with that at a depth of $10 \mathrm{~cm}$. By applying the correction factors obtained from the previous studies or limiting the application of the audit to a certain condition, TLSDs would be a candidate for the auditing dosimeter. In addition, several characteristics should be studied before applying it to the audit.

To use a TLSD for postal dosimetry, the thermal stability of its chemical composition and crystal structure must be verified to be high and a fading correction method must be established. Furthermore, the absorbed dose correction factor must be established under a certain condition and the uncertainty in dose measurement using a TLSD must be determined. ${ }^{(10)}$ In this study, we meet these requirements and evaluate the usefulness of postal output dosimetry for high-energy photon beams using a TLSD. 


\section{Materials and Methods}

\subsection{Cr-doped $\mathrm{Al}_{2} \mathrm{O}_{3}$ TLSD}

The TLSD is $0.7 \mathrm{~mm}$ thick and is composed of $99.5 \mathrm{wt} \% \mathrm{Al}_{2} \mathrm{O}_{3}$ and $0.05 \mathrm{wt} \% \mathrm{Cr}_{2} \mathrm{O}_{3}$. The density of the TLSD is $3.7 \mathrm{~g} / \mathrm{cm}^{3}$ and the effective atomic number is 11.13 . Figure 1 shows a photograph of a TLSD with an area of $80 \times 80 \mathrm{~mm}^{2}$.

\subsection{Thermal stability of TLSD chemical composition and crystal structure}

The TLSD was repeatedly heated and cooled to simulate repeated use. Changes in TLSD weight and temperature during repeated heating and cooling were measured using a differential thermal balance (Thermo Plus Evo, Rigaku Corporation). The reference sample was $\mathrm{Al}_{2} \mathrm{O}_{3}$. The weights of the reference and measurement samples were both $20 \mathrm{mg}$.

The temperature was increased at a rate of $30^{\circ} \mathrm{C} / \mathrm{min}$ from RT to $1000^{\circ} \mathrm{C}$. It was maintained at $1000{ }^{\circ} \mathrm{C}$ for $30 \mathrm{~min}$ and then reduced to RT at a rate of $15^{\circ} \mathrm{C} / \mathrm{min}$. This process was repeated 25 times. X-ray diffraction (XRD) analysis was performed using the Rigaku RINT2000. The XRD study showed corundum $\left(\alpha-\mathrm{Al}_{2} \mathrm{O}_{3}\right)$ for TLSDs. In this study, the changes in the chemical composition and crystal structure of the TLSD were evaluated by measuring the changes in TLSD weight and heat flow due to heating.

\subsection{TL characteristics of TLSD}

\subsubsection{Glow curve measurement system}

Figure 2 shows a schematic diagram of the glow curve measurement system. A TLSD with an area of $10 \times 10 \mathrm{~mm}^{2}$ was loaded into a heater in a dark box. The heater was heated to $400{ }^{\circ} \mathrm{C}$ at a rate of $0.13{ }^{\circ} \mathrm{C} / \mathrm{s}$ using a temperature controller (SCR-SHQ-A, SAKAGUCHI E.H VOC CORP.). The TL generated by heating was measured using a photon counting head (H11890-210,

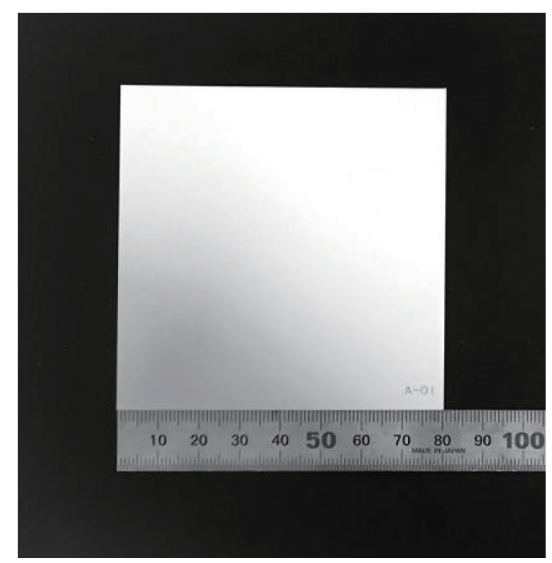

Fig. 1. Photograph of $\mathrm{Cr}$-doped $\mathrm{Al}_{2} \mathrm{O}_{3}$ TLSD. 


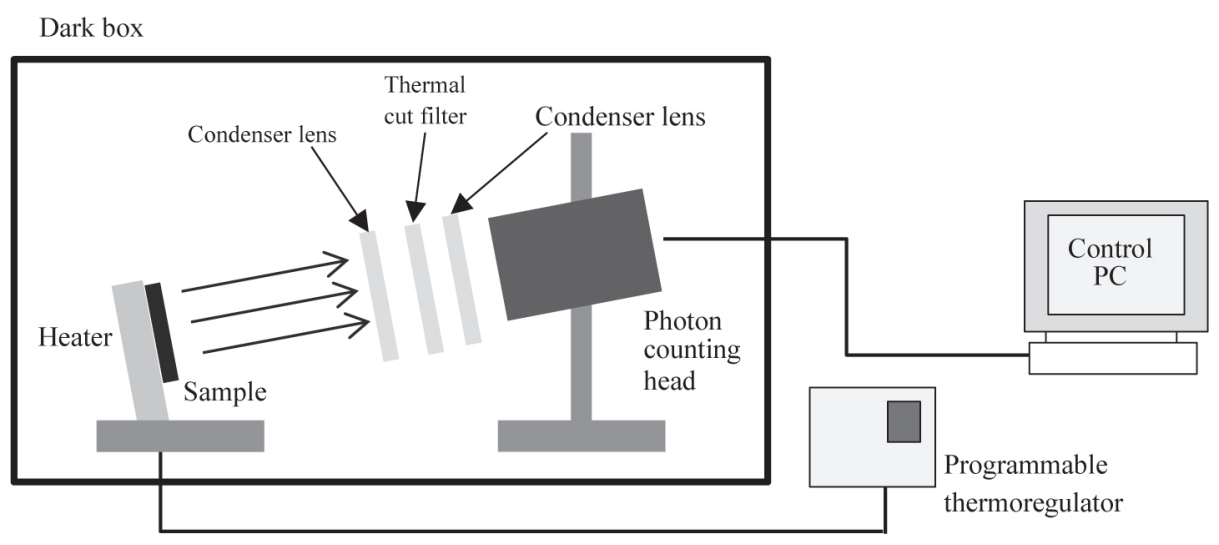

Fig. 2. Diagram of glow curve measurement system.

Hamamatsu Photonics K.K.) equipped with a focusing lens and a heat absorption filter. The measurement interval was $1 \mathrm{~s}$. The collected TL was processed on a control PC.

\subsubsection{Fading characteristics}

The TLSD was irradiated at 3 Gy using a linear accelerator (Clinac iX, Varian Medical Systems, Inc.) for radiotherapy. Figure 3 shows a diagram of the TLSD irradiation configuration. A TLSD with an area of $10 \times 10 \mathrm{~mm}^{2}$ was loaded at a depth of $10 \mathrm{~cm}$ into a stacked water-equivalent phantom (Tough Water, Kyoto Kagaku Co., Ltd.). The source-tosurface distance was $90 \mathrm{~cm}$. The radiation field size was $100 \times 100 \mathrm{~mm}^{2}$ and the X-ray energy was $10 \mathrm{MV}$. The TLSD was stored at $25{ }^{\circ} \mathrm{C}$ after irradiation. The TL intensity was measured using the glow curve measurement system. The TLSD was heated to $400{ }^{\circ} \mathrm{C}$ at a rate of $0.13{ }^{\circ} \mathrm{C} / \mathrm{s}$. After storage at $25^{\circ} \mathrm{C}$, glow curves were measured. The measurements were started 1 to $216 \mathrm{~h}$ after irradiation. The TL intensity was measured at various elapsed times using three TLSDs in total. The TL intensity from 40 to $400{ }^{\circ} \mathrm{C}$ was integrated into the glow curve. The relationship between the elapsed time until measurement and the TL intensity was derived. The fading correction equation was derived from the approximate curve.

\subsubsection{Measurement of X-rays using TLSD}

The TLSD was irradiated at 3 Gy using the irradiation configuration in Fig. 3. Eight measurements were taken. After storage at $25^{\circ} \mathrm{C}$, the measurements were started 22 to $26 \mathrm{~h}$ after irradiation. The glow curve of the TLSD was analyzed and the TL intensity from 40 to $400{ }^{\circ} \mathrm{C}$ was integrated into the glow curve. The TL reproducibility of the TLSD was evaluated. The TLSD was again irradiated from 1 to 3.5 Gy using a similar irradiation configuration. The relationship between the absorbed dose and the TL intensity was measured. 


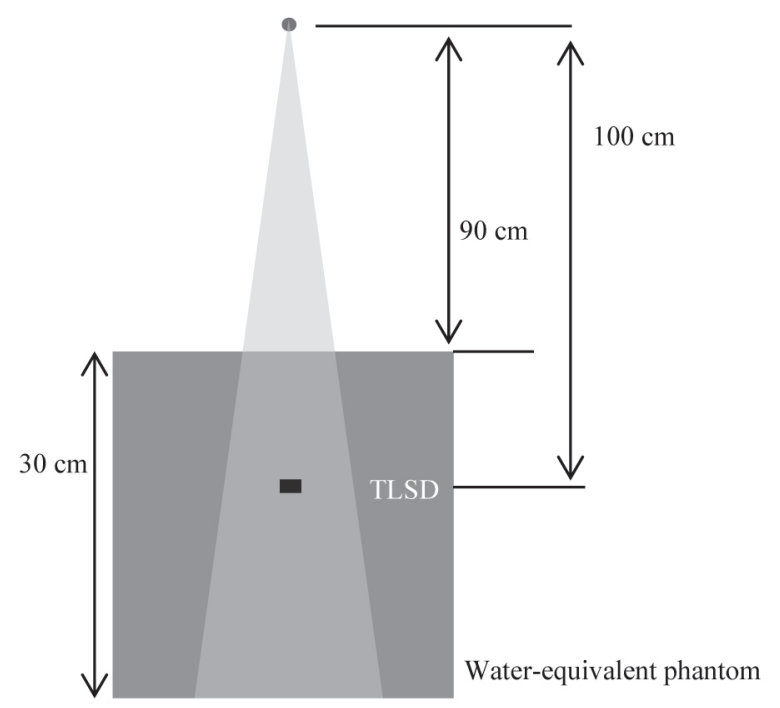

Fig. 3. Diagram of TLSD irradiation configuration for measuring fading characteristics.

\subsection{Dose distribution measurement using TLSD}

\subsubsection{TL distribution measurement system}

Figure 4 shows a diagram of the TL distribution measurement system. The TLSD was placed on three support needles fixed in a triangular configuration in a dark box. The hot plate, which was heated to $400{ }^{\circ} \mathrm{C}$ by the temperature controller, heated the TLSD by moving up to make contact with the TLSD. The generated TL was measured using a CMOS camera (ORCA ${ }^{\circledR}$-Flash 4.0 V2, C11440-22 CU, Hamamatsu Photonics K.K.) and processed on the control PC. The resolution of the acquired image was $0.05 \mathrm{~mm}$. Image analysis was performed using the image processing software ImageJ. The measurement time was $10 \mathrm{~s}$, which was the maximum exposure time of the CMOS camera. The measurement was repeated 25 times to ensure a sufficient readout time. The 25 th result was subtracted as the background. The other 24 results were summed to obtain the TL distribution.

\subsubsection{Derivation of TLSD absorbed dose correction factor}

The TL intensity can be converted to an absorbed dose by multiplying it by the TLSD absorbed dose correction factor $\left(C_{T L S D}\right)$ derived from the absorbed dose measured using the ionization chamber. The relationship between the TL intensity (TLI) and the absorbed dose $\left(D_{\text {chamber }}\right)$ can be expressed as

$$
D_{\text {chamber }}=T L I \times C_{T L S D} \times C_{\text {fading }}
$$




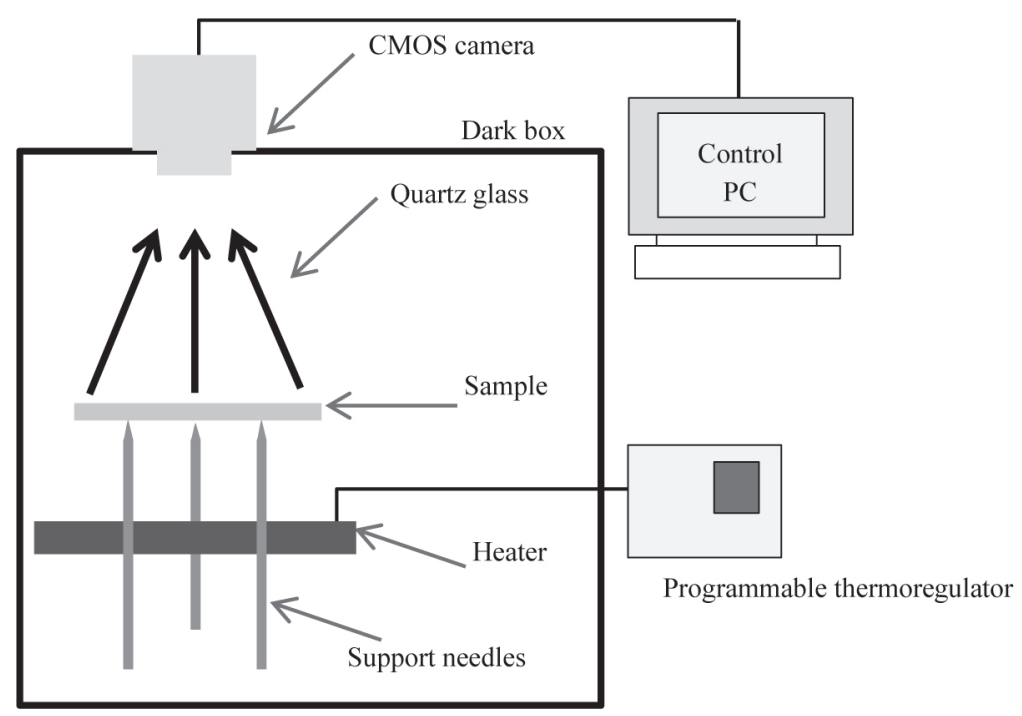

Fig. 4. Diagram of TL distribution measurement system.

where $D_{\text {chamber }}$ is the absorbed dose measured using the ionization chamber and $C_{\text {fading }}$ is the fading correction factor. $C_{T L S D}$ is calculated from the TL intensity obtained for irradiation with the reference dose.

In this study, $C_{T L S D}$ was calculated from the TL intensity obtained for irradiation with 2 Gy and the absorbed dose measured using the ionization chamber. Figure 5 shows a diagram of the TLSD irradiation configuration. A TLSD with an area of $80 \times 80 \mathrm{~mm}^{2}$ was placed at a depth of $10 \mathrm{~cm}$ in the water. The source-to-surface distance was $90 \mathrm{~cm}$. A linear accelerator similar to that mentioned in Sect. 2.3.2 was used. The X-ray energy was $10 \mathrm{MV}$ and the radiation field size was $50 \times 50 \mathrm{~mm}^{2}$. After irradiation, the TLSD was stored at $25{ }^{\circ} \mathrm{C}$. The measurements were started 95 to $100 \mathrm{~h}$ after irradiation. The TL intensity was corrected for fading.

$C_{T L S D}$ was calculated under a certain condition in this study. Therefore, several correction factors were included in $C_{T L S D}$. $C_{T L S D}$ was derived for two elements and the individual differences were evaluated.

\subsubsection{Absorbed dose distribution measurement using TLSD}

The TLSD was irradiated with 2 Gy and the TL distribution was measured using the TL distribution measurement system. Figure 6 shows a diagram of the TLSD irradiation configuration. A TLSD with an area of $80 \times 80 \mathrm{~mm}^{2}$ was placed at a depth of $10 \mathrm{~cm}$ in the water-equivalent phantom. The source-to-surface distance was $90 \mathrm{~cm}$. The X-ray energy and radiation field size were $10 \mathrm{MV}$ and $50 \times 50 \mathrm{~mm}^{2}$, respectively. The TLSD was stored at $25^{\circ} \mathrm{C}$ and the TL distribution was measured. The measurements were started 95 to $100 \mathrm{~h}$ after irradiation. Fading correction was applied.

The TL distribution was a corrected sensitivity distribution. ${ }^{(12)}$ The dose distribution was derived from Eq. (1) using the $C_{T L S D}$ determined in Sect. 2.4.2. ${ }^{(8)}$ 


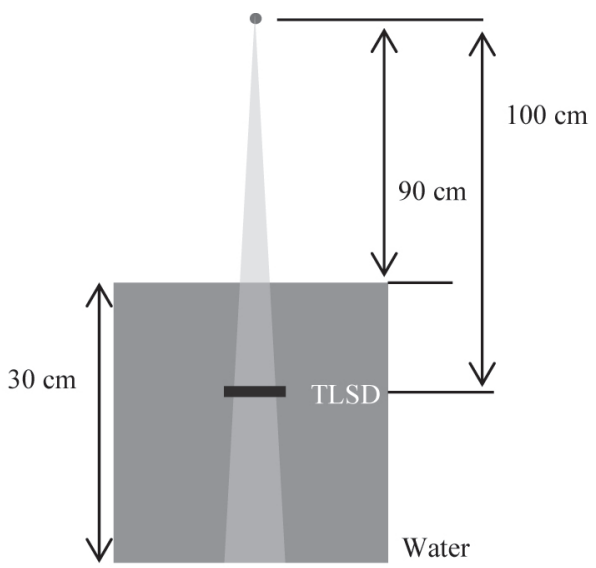

Fig. 5. Diagram of TLSD irradiation configuration for absorbed dose correction factor derivation.

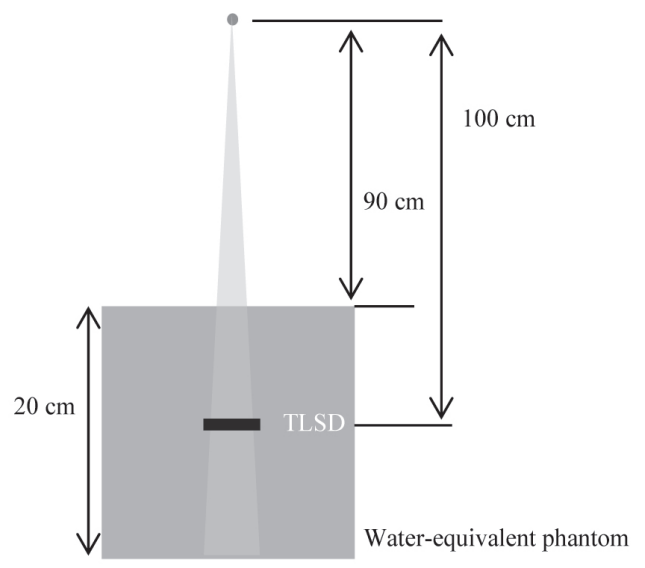

Fig. 6. Diagram of TLSD irradiation configuration for absorbed dose distribution measurement.

For the quality control of a radiation therapy system using the dose distribution, it is important to evaluate the invariance of the beam shape. For this evaluation, $80 \%$ of the beam area closest to the center is recommended. ${ }^{(6)}$ The dose distribution for the irradiation configuration similar to that in Fig. 6 was calculated using a radiation TPS (ECLIPSE, Ver. 13.6, Varian Medical Systems, Inc.). The results obtained using the radiation TPS and the measurement results for the TLSD were compared using the following equation:

$$
E=\frac{1}{N} \times \sum_{L=1}^{N}\left|\frac{T P_{L}-B P_{L}}{B P_{L}}\right| \times 100 \%
$$

where $N$ is the total number of measurement points used and $L$ is an index of measurement points. $T P_{L}$ and $B P_{L}$ are respectively the measurement results for the TLSD at an arbitrary position and the off-axis ratio calculated using the radiation TPS.

The dose distribution was also compared with that calculated using the TPS with the gamma method, which evaluates both dose differences and distance-to-agreement (DTA). The analysis software DD-System (DD IMRT version 12.42, R-Tec.inc, Japan) was used for verification. The acceptance criteria for dose differences and DTA were $2 \%$ and $2 \mathrm{~mm}$, respectively, at $80 \%$ of the beam area closest to the center.

\subsection{Uncertainty in absorbed dose distribution measurement using TLSD}

The factors that affect the uncertainty in the absorbed dose measurement using a TLSD can be divided into three types, namely, factors related to the TL intensity generated from the TLSD, factors related to the 2D TL distribution measurement system, and factors related to the irradiation system, including the linear accelerator. The extended relative standard uncertainty in the absorbed dose distribution measurement using the 2D TL distribution measurement system was obtained by deriving the uncertainty for each factor type. 


\subsubsection{Uncertainty in TL intensity of TLSD}

The uncertainties in the TL intensity are those from the TL reproducibility of a TLSD, fading calibration, sensitivity distribution calibration, and absorbed dose correction factor derivation.

TL reproducibility was calculated by the repeated measurement of the TL distribution measured on a TLSD placed in a water-equivalent phantom and irradiated with 2 Gy. Five measurements were taken. The irradiation scheme was similar to that described in Sect. 2.4.3.

The uncertainty in fading correction is the maximum difference between the measured value and the value calculated from the regression curve. The sensitivity correction coefficient $C_{S}(x, y)$ at the coordinates $(x, y)$ of the TL distribution obtained using the TLSD was derived as

$$
C_{S}(x, y)=\frac{D_{\text {chamber }}(x, y)}{T L I(x, y)},
$$

where $D_{\text {chamber }}(x, y)$ is the absorbed dose measured using the ionization chamber and $\operatorname{TLI}(x, y)$ is the TL intensity. The sampling interval of the ionization chamber dosimeter is larger than that of the TLSD. When $C_{S}(x, y)$ is calculated, $D_{\text {chamber }}(x, y)$ and $\operatorname{TLI}(x, y)$ are measured for uniform irradiation. Therefore, $D_{\text {chamber }}(x, y)$ for $\operatorname{TLI}(x, y)$ was calculated by interpolation. The volume average effect is not considered because of uniform irradiation. Therefore, the uncertainty in the 2D TL sensitivity distribution correction is the sum of the measurement reproducibility by the ionization chamber and the TL reproducibility. The uncertainty in the absorbed dose correction factor $C_{T L S D}$ depends on the TL reproducibility and the uncertainty in fading correction. Therefore, it was set as their sum.

\subsubsection{Uncertainty in TL distribution measurement system}

The factors related to the TL distribution measurement system are the measurement reproducibility of the CMOS camera and the variation of the background value when the heater temperature is $400{ }^{\circ} \mathrm{C}$. The measurement repeatability of the CMOS camera was taken as the sum of seven repeated measurements using a stabilized light source and the nominal value of the output stability.

Figure 7 shows a schematic diagram of the measurement system for measurement reproducibility with a stabilized light source. The stabilized light source comprises a spot light source (OPS-S20W-U, OPTEX FA Co., Ltd.) and a controller (OPPD-15, OPTEX FA Co., Ltd.). Measurement reproducibility was derived from five measurements of the stabilized light source placed at the TLSD installation position. The light intensity of the stabilized light source was the minimum output and the measurement time was $20 \mathrm{~ms}$.

A $10 \mathrm{~s}$ measurement using a CMOS camera without the TLSD was repeated 25 times using the heater heated to $400{ }^{\circ} \mathrm{C}$. The variation of the background of the CMOS camera was derived from the difference between the maximum and minimum values of the background. 


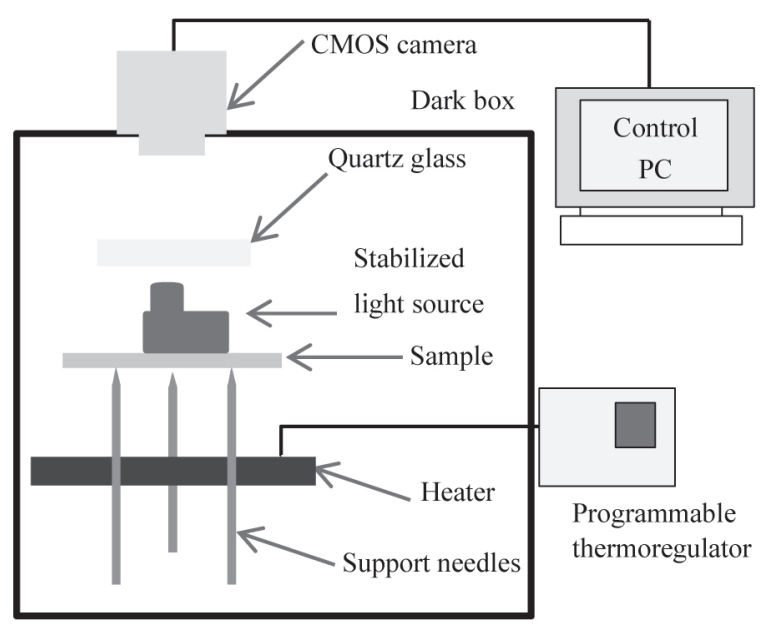

Fig. 7. Diagram of measurement reproducibility test with a stabilized light source.

\subsubsection{Irradiation system uncertainty}

The irradiation system uncertainty is related to the output reproducibility of the high-energy photon beam and the TLSD position. The output reproducibility of the high-energy photon beam was derived from five repeated measurements with an ionization chamber. The same phantom was used for all measurements and thus the phantom did not introduce uncertainty. The water-equivalent phantom loaded with the TLSD was placed at the irradiation center position using a laser indication point. The position error of the laser indication point was adjusted to be within $1 \mathrm{~mm}$. Therefore, the uncertainty due to phantom placement was $1 \mathrm{~mm}$.

\section{Results}

\subsection{Changes in TLSD weight and heat flow due to heating}

Figure 8 shows the relationship between the TLSD heating temperature and weight based on the second, sixth, eleventh, sixteenth, and twenty-first measurements. The TLSD weight gradually decreased immediately after heating. The TLSD weight decreased by about $1 \%$ at $1000{ }^{\circ} \mathrm{C}$ compared with that before heating. The TLSD weight returned to its original value before heating when the TLSD was cooled.

Figure 9 shows the relationship between the TLSD heating temperature and heat flow. The right vertical axis shows the electromotive force measured using a differential thermocouple. The change in TLSD heat flow is considered to be due to an endothermic reaction during heating. When the heating temperature reached $1000{ }^{\circ} \mathrm{C}$, the heat flow of the TLSD became constant. After cooling, the TLSD heat flow returned to its original value before heating.

The observed trends in the changes in TLSD weight and heat flow with heating temperature were the same for all measurements. The resolution of the equipment used for measuring the changes in weight was $0.1 \mu \mathrm{g}$. Therefore, the observed trends are not due to measurement variation. 


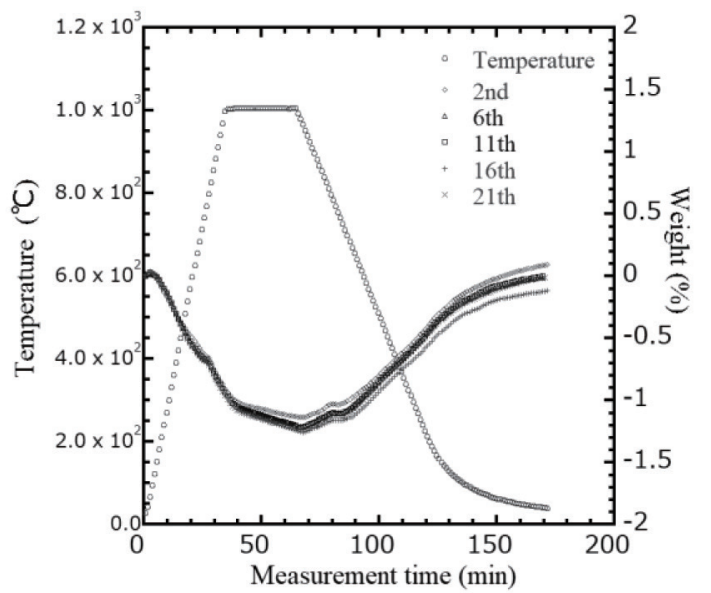

Fig. 8. Relationship between TLSD heating temperature and weight.

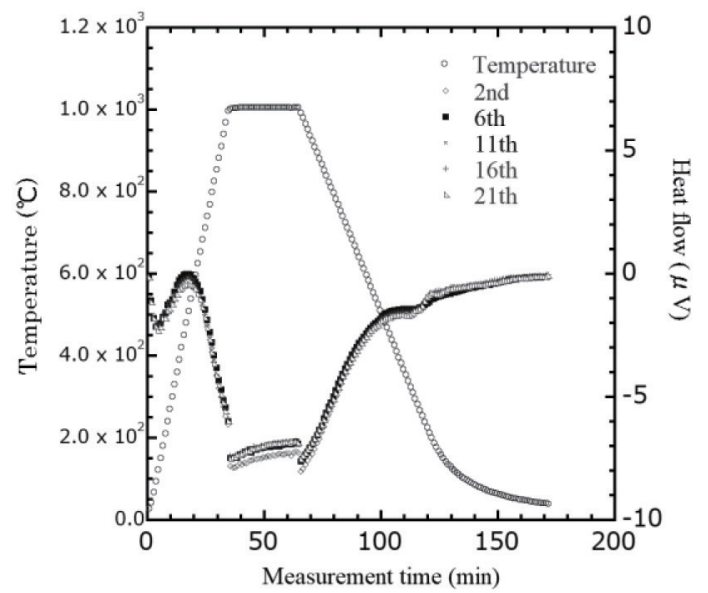

Fig. 9. Relationship between TLSD heating temperature and heat flow.

\subsection{TL characteristics of TLSD}

\subsubsection{Fading}

Figures 10(a), 11(a), and 12(a) show the relationship between the elapsed time after irradiation and the glow curve for the three TLSDs. The TL intensity decreased with increasing elapsed time. Figures 10(b), 11(b), and 12(b) show the glow curves normalized by their respective maximum values. The temperature and shape of the main peak of the glow curve did not change with elapsed time. However, the subpeak near $140{ }^{\circ} \mathrm{C}$ was observed only for the glow curve obtained $1 \mathrm{~h}$ after irradiation.

Figure 13 shows the relationship between the elapsed time and the TL intensity calculated from the integrated value of the glow curve. The TL intensity was normalized by that for an elapsed time of $100 \mathrm{~h}$. The TL intensity was sharply attenuated $24 \mathrm{~h}$ after irradiation. Gradual decay occurred after $100 \mathrm{~h}$. The TL intensity decay was determined to be about $1 \% / \mathrm{h}$ immediately after irradiation and $0.2 \% / \mathrm{h} 100 \mathrm{~h}$ after irradiation.

For TL measurement using the Cr-doped $\mathrm{Al}_{2} \mathrm{O}_{3}$ TLSD, the effect of fading can be minimized by setting the elapsed time to more than $100 \mathrm{~h}$. The fading correction factor $C_{\text {fading }}(x)$ obtained from the logarithmic approximation curve at an arbitrary elapsed time $x$ obtained from Fig. 13 is expressed as

$$
C_{\text {fading }}(x)=-0.36169 \times \log _{10}(x)+1.7234 \text {. }
$$

\subsubsection{TL response and dose linearity}

Figure 14 shows the glow curve of a TLSD irradiated with a $10 \mathrm{MV}$ X-ray beam. The glow curve has a main peak near $300{ }^{\circ} \mathrm{C}$ and a subpeak near $150{ }^{\circ} \mathrm{C}$. The TL reproducibility is the 


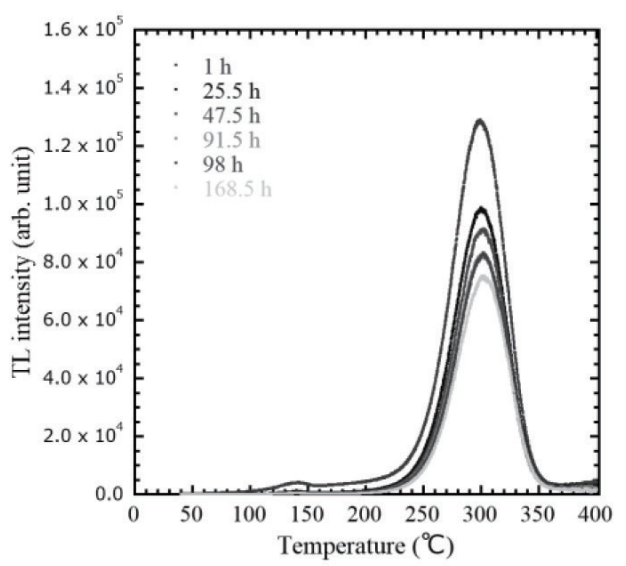

(a)

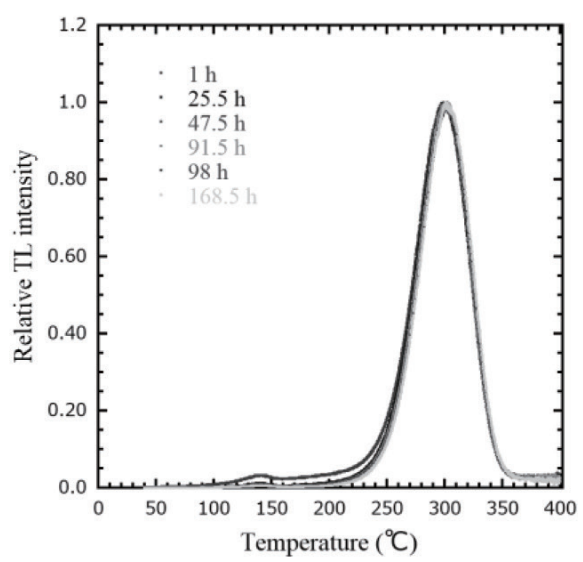

(b)

Fig. 10. Relationship between elapsed time and glow curve measured using TLSD No. 1. (a) Raw data and (b) data normalized by maximum value of each glow curve.

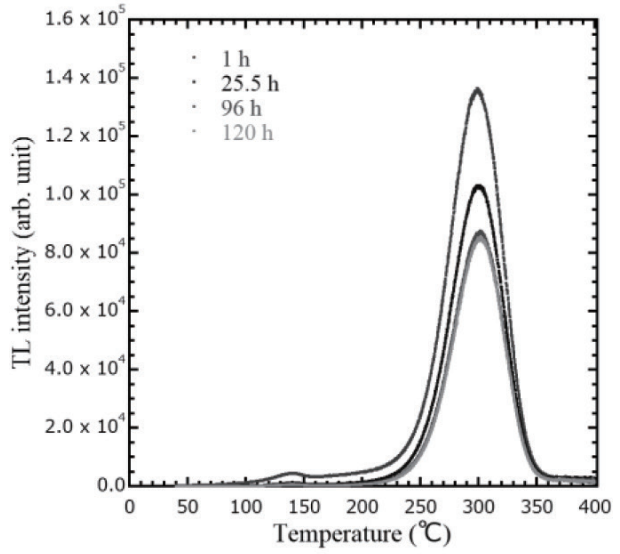

(a)

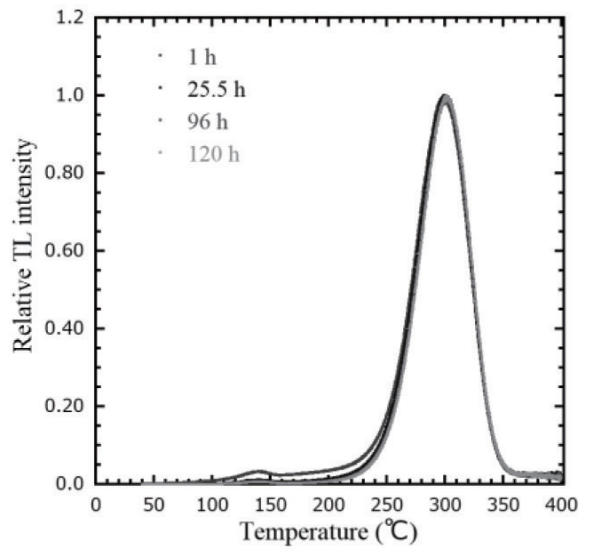

(b)

Fig. 11. Relationship between elapsed time and glow curve measured using TLSD No. 2. (a) Raw data and (b) data normalized by maximum value of each glow curve.

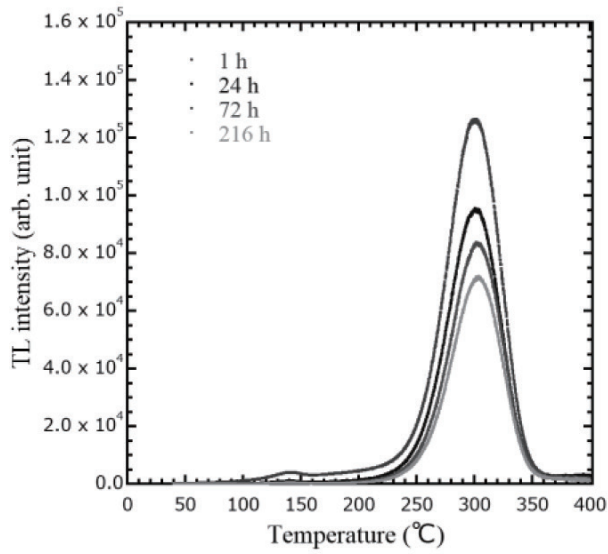

(a)

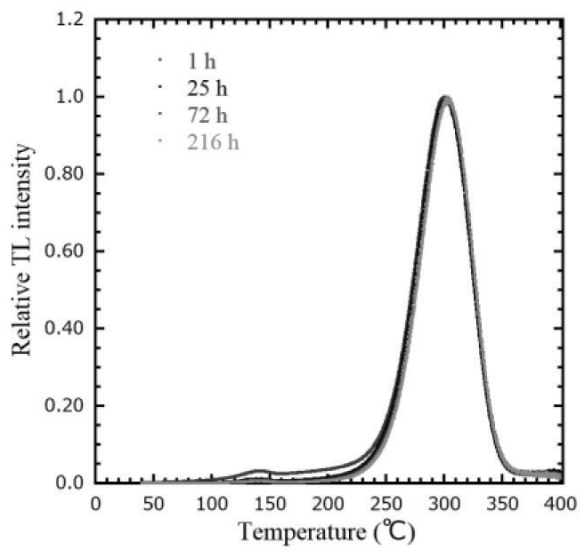

(b)

Fig. 12. Relationship between elapsed time and glow curve measured using TLSD No. 3. (a) Raw data and (b) data normalized by maximum value of each glow curve. 


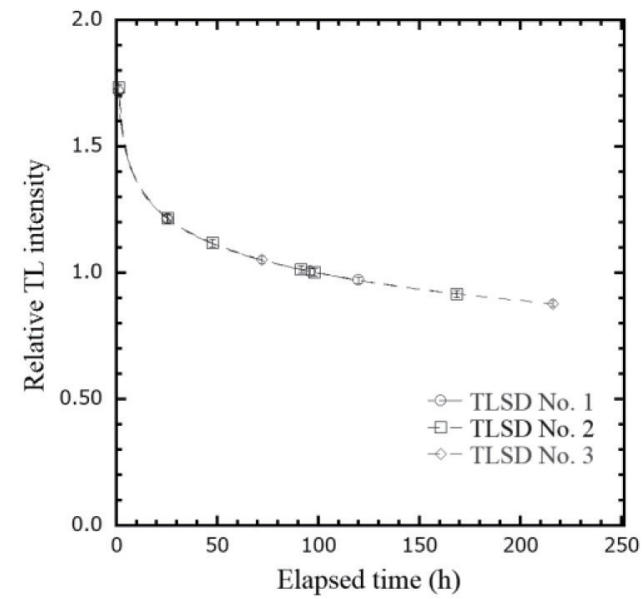

Fig. 13. Relationship between elapsed time and TL intensity. The error bars represent the coefficient of variation of the glow curve measurement.

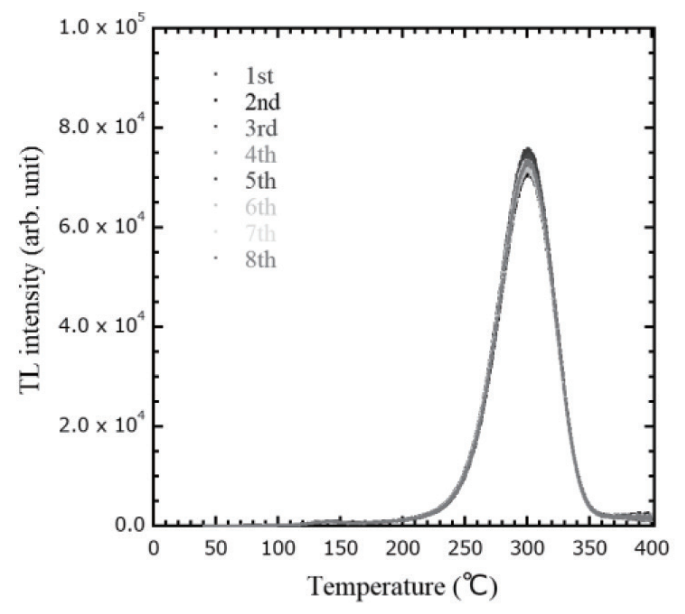

Fig. 14. Reproducibility of $10 \mathrm{MV}$ X-ray glow curve measured using TLSD.

coefficient of variation of the integrated TL intensity. The TL reproducibility of the TLSD was determined to be $2.03 \%$. When the fading correction was performed by applying Eq. (4) to the glow curve in Fig. 14, the TL reproducibility improved to $1.36 \%$.

Figure 15 shows the relationship between the absorbed and relative doses of the TLSD. The relative dose of the TLSD varies linearly with the absorbed dose. The coefficient of variation for each measurement point is $1.36 \%$, which is the TL reproducibility. The difference between the measured TL intensity for absorbed dose and that calculated from the approximate straight line obtained from the measurement was observed to be within $1.2 \%$ at maximum.

\subsection{Derivation of TLSD absorbed dose correction factor for TL intensity}

Figure 16 shows the TL distribution with a field size of $50 \times 50 \mathrm{~mm}^{2}$ measured using a TLSD with an area of $80 \times 80 \mathrm{~mm}^{2}$. The TLSD absorbed dose correction factor $C_{T L S D}$ was calculated using Eq. (1) based on the TL intensity at the center of the irradiation field. The variation coefficient of the TL intensity of the two TLSDs was determined to be $3.44 \%$. Therefore, the variation of $C_{T L S D}$ is at least greater than 3.44\%.

\subsection{Dose distribution measured using TLSD}

Figure 17(a) shows the dose distribution measured using the TLSD for a field size of $50 \times 50 \mathrm{~mm}^{2}$. The dose profile obtained along the horizontal line in (a) is shown in Fig. 17(b). The pixel size of the measured image is $0.05 \mathrm{~mm}$. The sampling interval of the dose profile calculated by the radiotherapy TPS is $1 \mathrm{~mm}$.

The dose profile measured using the TLSD agrees well with that calculated using the radiation TPS. The difference between the result calculated using Eq. (2) with the radiation 


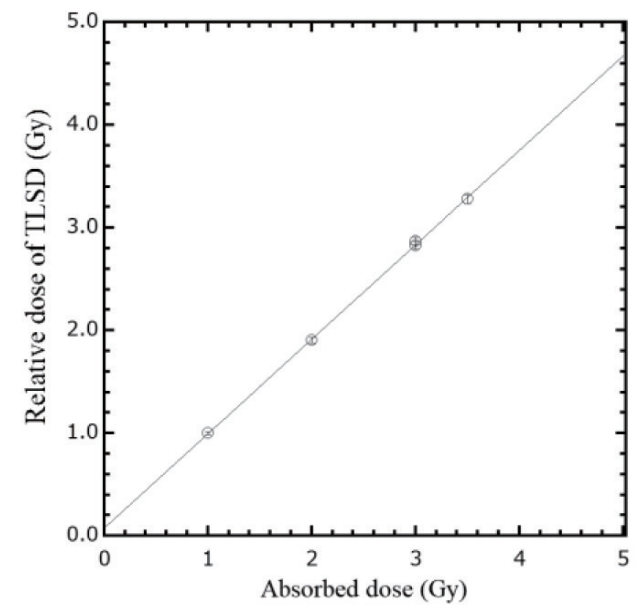

Fig. 15. Relationship between absorbed dose and TL intensity.

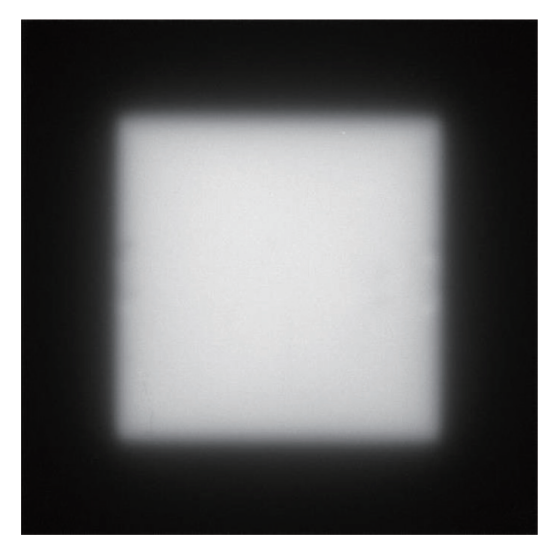

Fig. 16. TL distribution with field size of $50 \times 50 \mathrm{~mm}^{2}$.

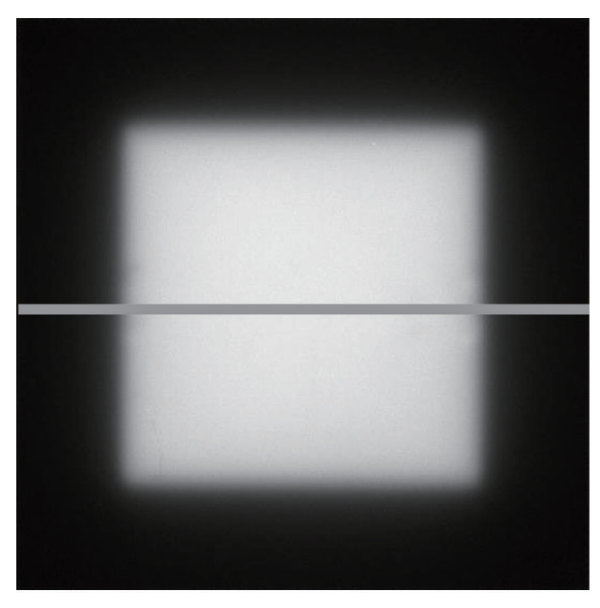

(a)

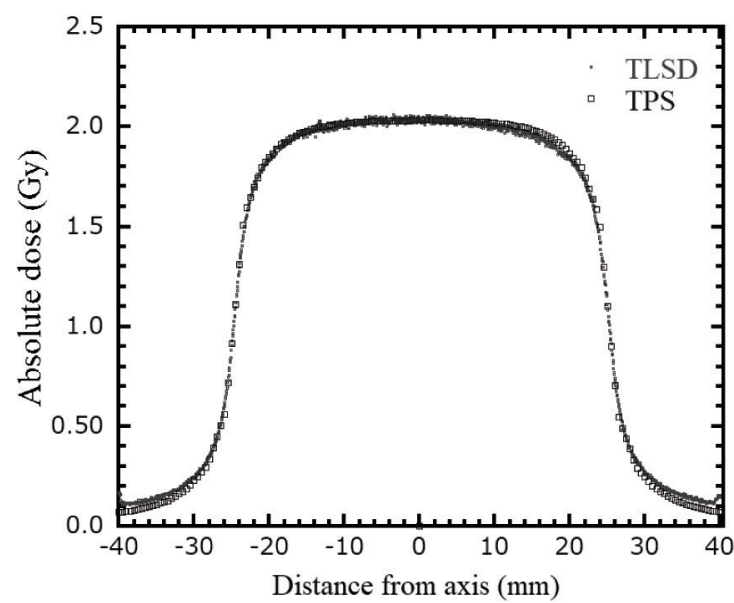

(b)

Fig. 17. Dose distribution measured using TLSD for field size of $50 \times 50 \mathrm{~mm}^{2}$. (a) Analysis range and (b) dose profile obtained along the horizontal line in (a).

TPS and the measurement result for the TLSD in $80 \%$ of the beam area closest to the center is $0.76 \%$. The path rate of gamma analysis is $98.42 \%$.

\subsection{Uncertainty in absorbed dose measurement using TLSD}

Table 1 shows the uncertainty budget for TLSD dosimetry. The causes of the uncertainty in TL intensity are the reproducibility of TL intensity, fading correction, TL distribution correction, and the uncertainty in absorbed dose correction factor derivation.

The reproducibility of TL intensity using the TL distribution measurement system, which is the coefficient of variation for five measurements, is $1.03 \%$. The relative standard uncertainty 
Table 1

Uncertainty budget for TLSD dosimetry. Uncertainty types A and B were calculated with and without statistical analysis of observations, respectively.

\begin{tabular}{|c|c|c|c|c|}
\hline & Factor & Evaluation method & $\begin{array}{l}\text { Uncertainty } \\
\text { type }\end{array}$ & $\begin{array}{c}\text { Relative standard } \\
\text { uncertainty (\%) }\end{array}$ \\
\hline \multirow{4}{*}{ TL intensity } & TL reproducibility & Repeated measurement & $\mathrm{A}$ & 0.47 \\
\hline & Fading & $\begin{array}{l}\text { Difference between calculation } \\
\text { and measurement }\end{array}$ & B & 0.14 \\
\hline & $\begin{array}{l}\text { 2D TL sensitivity } \\
\text { correction }\end{array}$ & $\begin{array}{l}\text { Sum of TL and X-ray output } \\
\text { reproducibilities }\end{array}$ & A & 0.47 \\
\hline & $\begin{array}{l}\text { Absorbed dose } \\
\text { correction factor }\end{array}$ & $\begin{array}{l}\text { Sum of TL reproducibility } \\
\text { and fading correction }\end{array}$ & $A+B$ & 0.49 \\
\hline \multirow{2}{*}{ CMOS camera } & $\begin{array}{l}\text { Measurement } \\
\text { reproducibility }\end{array}$ & $\begin{array}{l}\text { Measurement of stabilized light } \\
\text { source and nominal value }\end{array}$ & $A+B$ & 0.29 \\
\hline & $\begin{array}{l}\text { Background increase } \\
\text { due to heat radiation }\end{array}$ & $\begin{array}{l}\text { Difference between maximum } \\
\text { and minimum values }\end{array}$ & $\mathrm{B}$ & 0.15 \\
\hline \multirow{4}{*}{ Irradiation system } & Output reproducibility & Repeated measurement & A & 0.0002 \\
\hline & TLSD position & Maximum error of positioning laser & $\mathrm{B}$ & 0.005 \\
\hline & & \multicolumn{2}{|c|}{ Combined relative standard uncertainty (\%) } & 0.90 \\
\hline & & \multicolumn{2}{|c|}{ Extended relative standard uncertainty, $k=2(\%)$} & 1.80 \\
\hline
\end{tabular}

in the reproducibility of TL intensity is $0.47 \%$. In Eq. (3), the uncertainty in the TL distribution correction is obtained from the combination of TL and X-ray output reproducibilities. The X-ray output reproducibility is less than one-tenth of the TL reproducibility. The uncertainty in the absorbed dose correction factor obtained from the combination of TL reproducibility and fading correction is $0.49 \%$.

The nominal value of the output stability of the stabilized light source of the CMOS camera is $0.5 \%$ and the uncertainty is $0.29 \%$. The uncertainty for the repeated measurements of the stabilized light source is negligible. The cause of measurement uncertainty for the CMOS camera depends on the light emission accuracy of the stabilized light source. The relative standard uncertainty in background variation is $0.15 \%$ for the heater heated to $400{ }^{\circ} \mathrm{C}$.

The cause of the uncertainty in the irradiation system is less than one-tenth of the uncertainty in the measurement reproducibility of the CMOS camera. Therefore, the extended relative standard uncertainty is $1.80 \%$ under a certain condition for inclusion coefficient $k=2$.

\section{Discussion}

\subsection{Fading}

Figures 10-12 show that the shape of the glow curve changes with elapsed time before measurement. This change is due to the difference between the thermoluminescent component that decays immediately after irradiation and the component that decays after $24 \mathrm{~h}$ or more. The Cr-doped $\mathrm{Al}_{2} \mathrm{O}_{3}$ TLSD has glow curves with peaks near 300 and $140{ }^{\circ} \mathrm{C}$. 
The fading in the TL measurement can be reduced by reducing the elapsed time. However, measurement immediately after irradiation is impossible for postal dosimetry. Correcting the attenuation with elapsed time improves measurement accuracy. This correction is most effective when the attenuation per unit time is small. Therefore, for TL measurement using a TLSD, the TL intensity should be measured after more than $24 \mathrm{~h}$ have passed. It is important to set an appropriate elapsed time for measuring TL intensity fading versus elapsed time.

When the fading correction was performed by applying Eq. (4) to the glow curve in Fig. 14, the TL reproducibility improved to $1.36 \%$. Therefore, measurement accuracy can be improved by using the fading correction factor calculated from measurement data.

Fading correction based on the results measured in this study required data to be acquired. If the fading curve obtained by the measurement can be analyzed on the basis of TL theory, the fading correction can be easily performed using the activation energy required to liberate a trapped charge carrier.

\subsection{Thermal stability of TLSD chemical composition and crystal structure}

Figures 8 and 9 show that the weight of the TLSD decreases owing to an endothermic reaction during heating. The weight decreased by about $1 \%$ after heating from RT to $1000{ }^{\circ} \mathrm{C}$. The melting point of the reference sample of $\mathrm{Al}_{2} \mathrm{O}_{3}$ is $2072{ }^{\circ} \mathrm{C}$. The melting point of the TLSD, which is $\mathrm{Cr}$-doped $\mathrm{Al}_{2} \mathrm{O}_{3}$, is considered to be lower than that of $\mathrm{Al}_{2} \mathrm{O}_{3}$. It is considered that the TLSD weight changes owing to an endothermic reaction during heating to a temperature near the melting point. This reaction is likely due to water molecules bound to the TLSD. No endothermic peak was observed because the heating temperature did not reach the melting point.

The weight of the TLSD changed after heating. This change was reversible with cooling. Furthermore, the change in weight was observed after repeated heating. Figure 14 shows that the change in weight caused by heating does not affect the TL characteristics. Therefore, the material structure of the TLSD is not changed by heating.

\subsection{TLSD absorbed dose calibration factor for TLSD}

There is variation in the TL distributions obtained using TLSDs due to slab processing. The absorbed dose calibration factor should be determined for each TLSD. Figure 15 shows that the linearity of the TLSD is good in the dose range of the X-ray beam used in radiotherapy (about 3 Gy). Therefore, the absorbed dose calibration factor obtained from the reference dose can be used for the dose distribution measurement from 0.1 to 4 Gy used in this study.

\subsection{Dose distribution measurement using TLSD}

For the quality control of a radiation therapy system, it is important to evaluate the constancy of the beam profile at the time of system installation. ${ }^{(6)}$ The dose profile shown in Fig. 17(b) shows that the average difference between the value measured using the TLSD and 
the calculated value of the radiation TPS is $0.76 \%$. It is recommended that the beam profile constancy be less than $1 \%$ compared with that in the case of the installation. Moreover, the dose distribution was within the tolerance level in gamma analysis. Therefore, the TLSD has sufficient measurement accuracy for the quality control of the beam profile.

In this study, dose distribution measurement accuracy was evaluated assuming the thirdparty evaluation of a flat profile. In a previous study, the accuracy was evaluated without considering the fading of intensity-modulated radiotherapy. ${ }^{(8)}$ In the future, the third-party evaluation of intensity-modulated radiotherapy will be possible by postal dosimetry.

\subsection{Uncertainty in absorbed dosimetry using TLSD}

Table 1 shows that the relative standard uncertainty in TL for the TLSD is $0.47 \%$. This is the TL reproducibility of the Cr-doped $\mathrm{Al}_{2} \mathrm{O}_{3}$ of the TLSD. The uncertainty in TL is also related to absorbed dose correction factor derivation and 2D TL sensitivity correction factor derivation. Furthermore, the uncertainty in the absorbed dose correction factor derivation also depends on the uncertainty due to fading correction. Therefore, improving the TL reproducibility for the TLSD can reduce the uncertainty in TL measurement.

Furthermore, the relative standard uncertainty in the CMOS camera using the stabilized light source was determined to be $0.29 \%$. This result is due to the nominal value of the luminance fluctuation of the stabilized light source. The uncertainty in measurement using the CMOS camera is affected by the error of the stabilized light source, which determines the measurement accuracy of the detector. The variation of the CMOS camera measured using the stabilized light source is less than one-tenth of the nominal value of the luminance fluctuation. Therefore, the uncertainty for the repeated measurements of the stabilized light source is negligible. The measurement accuracy of the CMOS camera is higher than the measurement uncertainty measured in this study.

The relative standard uncertainty in the background variation was determined to be $0.15 \%$ for the heater heated to $400{ }^{\circ} \mathrm{C}$. This is considered to be the effect of heat radiation during TLSD heating. The extended relative standard uncertainty in absorbed dosimetry using the TLSD under a certain condition was determined to be $1.80 \%$. This is close to the uncertainty in postal dosimetry using a glass dosimeter in Japan. ${ }^{(13,14)}$

\section{Conclusions}

In this study, we evaluated the material stability of a TLSD, derived a TL decay correction method and a TLSD absorbed dose correction factor, and measured the uncertainty in dose distribution measurement using a TLSD for postal dosimetry.

The TLSD was found to have high material stability and can thus be used repeatedly. Accuracy can be improved using fading correction. The dose distribution measurement using a TLSD under a certain condition has sufficient accuracy for the quality control of a radiotherapy system. The extended relative standard uncertainty in the entire system in a field size of $50 \times 50 \mathrm{~mm}^{2}$ for a $10 \mathrm{MV}$ X-ray beam was determined to be $1.80 \%$. The TLSD has the 
potential to perform postal dosimetry audit under a certain condition. To conduct measurements at various conditions, it is necessary to consider correction methods that consider beam energy. Furthermore, a measurement experiment that uses delivery should be conducted to verify accuracy. The TLSD is a 2D measurement device that can be used for postal dosimetry audit.

\section{References}

1 D. Ciardo, D. Alterio, and B. A. Jereczek-Fossa: Phys. Med. 31 (2015) 1015.

2 A. W. Boyd and H. H. Eisenlohr: Med. Phys. 10 (1983) 491.

3 H. H. Eisenlohr and S. Jayaraman: Phys. Med. Biol. 22 (1977) 18.

4 H. Mizuno, T. Kanai, and Y. Kusano: Radiother. Oncol. 86 (2008) 258.

5 F. Araki, N. Moribe, and Y. Yamashita: Med. Phys. 31 (2004) 1980.

6 AAPM Task Group No. 142: Med. Phys. 36 (2009) 4199.

7 K. Shinsho, D. Maruyama, and S. Yanagisawa: Sens. Mater. 30 (2018) 1591.

8 S. Yanagisawa, K. Shinsho, and M. Inoue: Radiat. Meas. 109 (2017) 326.

9 S. Yanagisawa, D. Maruyama, and K. Shinsho: to be published in Sens. Mater.

10 J. Izewska, H. Svensson, and G. Ibbott: Pro. Int. Symp. Standards and Codes of Practice in Medical Radiation Dosimetry IAEA-CN-96/76 (IAEA, 2002) 139.

11 C. Y. Leong, H. Wagiran, and A. K. Ismail: J. Radioanal. Nucl. Chem. 307 (2016) 229.

12 K. Shinsho, Y. Koba, and G. Wakabayashi: Radiat. Meas. 62 (2014) 15.

13 T. H. Kirby, W. F. Hanson, and D. A. Johnston: Med. Phys. 19 (1992) 1427.

14 H. Mizuno, A. Fukumura, and M. Fukahori: Med. Phys. 41 (2014) 112. 